\title{
DESENVOLVIMENTO DE METODOLOGIA PARA PREVISÃO DO FENÔMENO DE VIBRAÇÕES DECORRENTES DA INTERFERÊNCIA ENTRE EIXOS DE UM TRATOR COM TRAÇÃO DIANTEIRA AUXILIAR (TDA) ${ }^{1}$
}

\author{
DEVELOPMENT OF METHODOLOGY TO PREDICT POWER HOP OF THE AXLE \\ INTERFERENCE OF A FRONT WHEEL ASSIST TRACTOR (FWA)
}

\author{
José Fernando Schlosser ${ }^{2}$ Luis Márquez ${ }^{3}$ Pilar Linares $^{3}$
}

RESUMO

Foi desenvolvido um ensaio de campo com o objetivo de avaliar as condições da ocorrência do fenômeno do "galope" do trator, que consiste em vibrações de baixa freqüência e grande amplitude. Este fenômeno físico é muito comum nos tratores de tração integral, principalmente nos que possuem pneus de diferentes diâmetros no eixo dianteiro $e$ traseiro. Para provocar o "galope", determinaram-se diferentes condições de distribuição de peso, pressão interna dos pneus e da magnitude da carga na barra de tração. Ao final dos ensaios, determinaram-se as condições de ocorrência do fenômeno por meio de uma metodologia bastante simples em função dos raios dos pneus. $O$ método utilizado para a determinação das condições de ocorrência do galope foi plenamente satisfatório, pois pôde prever com bastante precisão as condições em que iria ocorrer o fenômeno.

Palavras-chave: tração dianteira auxiliar, galope, tratores.

\section{SUMMARY}

A field experiment was carried out to evaluate the conditions for the appeareance of the power hope phenomenon. It consists in low frequency vibrations and great amplitude. This physical phenomenon is very common in FWA tractors, mainly with unequal tires diameter in front and rear axle. To promote power hope, different weight distribution conditions, different tire pressure and different drawbar force were used. Power hope occurence conditions were determined using simple methodology, which uses tire radio in the calculations.

Key words: front wheel assist, power hope, tractors.

\section{INTRODUÇÃO}

Nos tratores de tração nas quatro rodas, tanto de rodas iguais como de tamanho diferente nos eixos dianteiro e traseiro, surgem problemas ao tentar compatibilizar o empuxo de rodas que trabalham sobre solos de diferente natureza (compactado e não compactado), mais ainda quando estas são de diferente dimensões. As soluções adotadas para alcançar os rendimentos adequados se baseiam principalmente em modificar a relação cinemática das rodas de cada eixo, tanto por construção, como pela variação do raio dinâmico dos pneus de cada eixo, os quais são influenciados pela distribuição de massa que se coloca no trator pelas pressões internas utilizadas.

Autores como SRIVASTAVA et $\boldsymbol{a l}$. (1993) descrevem assuntos relacionados à tração e, entre os tratores com tração dianteira auxiliar (TDA), em que a velocidade das rodas dianteiras é pouco maior que as traseiras. Quando a ligação entre os eixos é rígida, haverá obrigatoriamente uma relação invariável entre as velocidades angulares dos eixos. Nesta configuração, pode produzir-se uma discordância cinemática entre eles, fazendo com que a velocidade teórica entre rodas possa variar, ainda que as velocidades reais de avanço nos eixos dianteiro e traseiro devam ser iguais.

No projeto do trator, os técnicos colocam uma relação constante entre os eixos, mas a

\footnotetext{
${ }^{1}$ Parte da tese de doutorado do primeiro autor, Universidad Politécnica de Madrid, Espanha.

${ }^{2}$ Engenheiro Agrônomo, Doutor, Professor Titular do Departamento de Engenharia Rural, Centro de Ciências Rurais, Universidade Federal de Santa Maria, Campus Universitário Camobi, 97.105-900, Santa Maria, RS. E-mail: schlosse@ccr.ufsm.br. Autor para correspondência.

${ }^{3}$ Engenheiro Agrônomo, Doutor, Professor Titular do Departamento de Ingenieria Rural, ETSIA, UPM, Madrid, Espanha.
} Recebido para publicação em 21.08.00. Aprovado em 11.04.01 
velocidade das rodas pode variar em função de vários fatores, entre eles, a transferência de peso, o desgaste diferenciado dos pneus e a pressão interna. A discordância cinemática também aparece quando o trator está desenvolvendo seu trabalho em uma trajetória curva.

Com referência ao fenômeno do "galope" do trator (wind-up), RACKHAM \& BLIGHT (1985) o definem como a interferência entre duas rodas de diferentes diâmetros conectadas rigidamente a uma mesma fonte de transmissão de potência e obrigadas a girar com a mesma velocidade mantendo contato com a superfície do terreno. Geralmente este efeito pode provocar severos desgastes nos pneus. Quando os raios dinâmicos, em condição de trabalho de tração, não são iguais, afetam as velocidades periféricas das rodas motrizes, ocorre o fenômeno do "wind-up" ou "power hop", segundo outros autores. As diferenças nos raios podem produzir-se por diversos motivos, entre os quais os autores destacam, a distribuição dinâmica das massas sobre os eixos, as pressões internas, os desgastes e as características particulares dos pneus.

Em trabalho sobre rendimento de tratores com tração dianteira auxiliar, BASHFORD (1985) teve como objetivo investigar a influência da força de tração e a eficiência em tração de um trator agrícola com TODA. Ao trabalhar com diferentes relações de velocidades entre as rodas traseira e dianteira, diferentes condições de peso e com o acionamento ou não da tração dianteira auxiliar, trabalhou com um trator em duas condições de solo distintas, concreto e solo argiloso lavrado. Em solo agrícola a $4 \mathrm{~km} / \mathrm{h}$, concluiu que a tração bruta não diferiu nos tratamentos com relações cinemáticas maiores de 1 e eram superiores ao tratamento com a relação cinemática de 0,97 ao redor de $10 \%$. Em termos de eficiência em tração, os valores máximos foram alcançados com relação cinemática de 1,01, entre os valores de 30 e $35 \%$ do peso total no eixo dianteiro. Percentagens de peso dianteiro superiores a este valor provocavam diminuição de eficiência nas relações de 1,01 e 1,07, enquanto com as demais relações cinemáticas $(0,97$ e 1,13$)$ o ponto de mínima eficiência correspondia a esta faixa entre 30 e $35 \%$ do peso total no eixo dianteiro.

Esta análise teórica indica que, para um veículo de tração nas quatro rodas, com acoplamento rígido entre os eixos, será possível alcançar a máxima eficiência em tração quando a velocidade teórica das rodas dianteiras e traseiras forem iguais, permitindo assim, patinamento similar nas rodas nos dois eixos. Nesta condição, a distribuição do empuxo é otimizada automaticamente, de forma que a distribuição de peso não afetará o rendimento.
O objetivo deste trabalho foi encontrar uma metodologia de fácil aplicação que proporcionasse a detecção de condições em que o fenômeno do galope pudesse ocorrer, utilizando artifícios simples de cálculos e facilidade na obtenção de dados. Comparando-se as previsões calculadas com o ocorrido no campo e as variações de distribuição de peso, pressões internas adotadas e os níveis de carga impostas à barra de tração, podese determinar as condições de trabalho que evitariam o fenômeno.

\section{MATERIAL E MÉTODOS}

Para a comprovação do método, foi utilizado um trator de rodas marca John Deere, modelo 6400, com eixo dianteiro motriz e transmissão tipo "PowrQuad". O trator estava equipado com um motor Diesel, com turbocompressor, 4 cilindros e volume de $4530 \mathrm{~cm}^{3}$. A potência máxima na tomada de potência é de $73 \mathrm{~kW}(100 \mathrm{CV})$ a $2300 \mathrm{rev} / \mathrm{min}$. Os pneus que equipavam este trator eram Michelin 16.9 R 24 BIB $\mathrm{x}$ M18 T2 134 A8 131B nas rodas dianteiras e Firestone Radial 70018.4 R 38146 A8 143B nas traseiras. Foi utilizada uma instrumentação eletrônica de aquisição de dados formada por um conjunto de sensores (velocidade das rodas, velocidade do veículo (radar) e célula de carga (eixo dinamométrico), conexões, cabos e um condicionador de sinais, junto com um programa de aquisição de dados, que possibilitou seu armazenamento em um computador.

$\mathrm{O}$ estudo se desenvolveu em duas fases distintas: solo firme de textura franco arenosa (resteva de trigo) umidade gravimétrica de 4,12\% (0 a $15 \mathrm{~cm}$ ) e em solo preparado do mesmo tipo com $7,58 \%$ de umidade gravimétrica e baseava-se na variação de condições de utilização do trator que induzisse, em alguns casos, o aparecimento do fenômeno vibratório. As condições do experimento estão apresentadas na tabela 1.

A relação cinemática foi calculada a partir dos raios estáticos das rodas e da relação entre as velocidades angulares de cada eixo do trator.

Para determinação das condições em que ocorre o "galope" (vibrações), foi proposto uma metodologia baseada nos conceitos de LINARES (1996). A metodologia prevê o reconhecimento de raios de referência, que são medidos sem carga, pela distância percorrida no campo e os raios dinâmicos, de cada condição, solo firme e solo preparado, medidos em trabalho de tração. Com estes, determina-se a relação entre raios e a relação entre velocidades periféricas das rodas, utilizadas para 
Tabela 1 - Condições do experimento demonstrado pelos fatores e seus níveis utilizados no experimento.

\begin{tabular}{|c|c|c|c|}
\hline & FATOR P & FATOR D & FATOR C \\
\hline & Pressão interna & Distribuição de peso & Carga na barra \\
\hline Nível 1: & $\begin{array}{c}100 \mathrm{kPa} \text { nos pneus } \\
\text { dianteiros, } 220 \mathrm{kPa} \text { nos } \\
\text { pneus traseiros }\end{array}$ & $\begin{array}{l}49 \% \text { do peso total no eixo } \\
\text { dianteiro, } \\
51 \% \text { do peso total no eixo } \\
\text { traseiro }\end{array}$ & $\begin{array}{l}\text { Trator freio a } 2000 \\
\text { rev/min na } 2^{\mathrm{a}} \text { marcha }\end{array}$ \\
\hline Nível 2: & $\begin{array}{c}160 \mathrm{kPa} \text { nos pneus } \\
\text { dianteiros, } 160 \mathrm{kPa} \text { nos } \\
\text { pneus traseiros }\end{array}$ & $\begin{array}{l}45 \% \text { do peso total no eixo } \\
\text { dianteiro, } \\
\begin{array}{l}55 \% \text { do peso total no eixo } \\
\text { traseiro }\end{array}\end{array}$ & $\begin{array}{l}\text { Trator freio a } 2000 \\
\text { rev/min na } 1^{\mathrm{a}} \text { marcha }\end{array}$ \\
\hline Nível 3: & $\begin{array}{c}220 \mathrm{kPa} \text { nos pneus } \\
\text { dianteiros, } 160 \mathrm{kPa} \text { nos } \\
\text { pneus traseiros }\end{array}$ & $\begin{array}{l}41 \% \text { do peso total no eixo } \\
\text { dianteiro, } \\
\begin{array}{l}59 \% \text { do peso total no eixo } \\
\text { traseiro }\end{array}\end{array}$ & $\begin{array}{l}\text { Trator freio em marcha } \\
\text { lenta na } 1^{\mathrm{a}} \text { marcha }\end{array}$ \\
\hline
\end{tabular}

estabelece por meio das relações de transmissão entre os dois diferenciais. Geralmente esta relação de velocidades é maior que 1 , a favor do eixo dianteiro, que gira mais rápido que o traseiro. $\mathrm{O}$ segundo fator é o próprio raio das rodas, que pode ser alterado pelo usuário, por meio da troca dos pneus e/ou pela variação da distribuição de peso e/ou da pressão interna dos mesmos.

Neste trabalho, obtiveramse distintas relações cinemáticas estáticas por meio das diferentes combinações de pressão interna entre os pneus dos dois eixos. Em dois níveis do fator "pressão interna",

determinar o patinamento relativo entre as rodas dianteiras e traseiras que serve para estimar as condições de aparecimento do fenômeno do "galope" do trator.

Assim, depois de medirem-se os raios de referência e em tração, determina-se a relação dinâmica entre raios $(B r)$. Tendo-se a relação dinâmica entre raios, pode-se calcular a relação entre velocidades das rodas (Er), com o valor da relação cinemática (a), que, para o trator testado, era de 1,3828 .

$$
E r=a . B r
$$

Assim como se calcula a relação entre velocidade das rodas de referencia, $E r$, pode-se calcular a relação entre rodas em condição de tração nas duas condições de solo $(E q)$. O patinamento será o quociente entre o $E r$ e o $E q$.

Utilizou-se um delineamento estatístico fatorial de três fatores e 3 níveis de cada fator, isto é $3 \times 3 \times 3$. O delineamento estatístico utilizado foi de blocos ao acaso. Havia três blocos, cada um deles com uma série completa de tratamentos.

A análise estatística dos dados foi realizada com o programa estatístico SAS (Statistical Analisys System), utilizando correlação entre as variáveis, pelo Coeficiente de Correlação de Pearson, a análise da variância e a estimativa dos coeficientes de regressão entre as variáveis.

\section{RESULTADOS E DISCUSSÃO}

A relação cinemática entre os eixos do trator depende de dois fatores: a relação de velocidade angular entre os eixos e os raios das rodas dianteira e traseira do trator. O primeiro fator é fixo e depende do projeto do fabricante que o mantinham-se pressões diferentes nos pneus de cada eixo, e em um se mantinha a pressão recomendada pelo fabricante do trator $(160 \mathrm{kPa})$ em todos os pneus. Esta combinação de pressões permitiu conseguir diferentes raios estáticos das rodas, o que ocasionou diferentes relações cinemáticas.

As relações variaram entre 0,975 e 1,088, isto é, $-2,5 \%$ e $8,8 \%$. Com a primeira, a relação cinemática é de atraso, as rodas traseiras têm velocidade teórica mais alta que as dianteiras e, no segundo caso, é de adianto, as rodas dianteiras têm a velocidade teórica mais alta que as traseiras. Em geral, conseguiu-se um escalonamento de relações estáticas de $-2,5 \% ;-2,3 \% ; 0,6 \% ; 2,8 \% ; 4,4 \% ; 5,1 \%$; $6,9 \% ; 8,5 \% ; 8,8 \%$ sendo duas de atraso (com sinal negativo) uma de igualdade e seis de adianto cinemático estático. A condição estática supõe que a determinação se realize utilizando o raio estático sob carga, com o trator parado sobre uma superfície plana e regular, com as características de pressão interna e distribuição do tratamento a que se está fazendo referência.

Como superfície de referência, foi utilizada a condição de solo duro (resteva). Nesta situação, os raios utilizados para o cálculo da relação cinemática eram os raios dinâmicos de referência. Os resultados apresentaram diferença entre o maior e o menor valor, de $11,3 \%$ para $2,8 \%$. As relações cinemáticas dinâmicas se aproximaram a $1 \mathrm{e}$, nesta condição de trabalho, casoexerça tração, ocorre uma uma redução nos valores da relação cinemática em relação à estática para todos os tratamentos e uma absorção das diferenças pelos pneus, induzindo a tal comportamento. Outra forma de calcular a relação cinemática é a condição dinâmica sob tração, isto é, calculada a partir dos dados das provas com tração e com as condições que supõe cada tratamento. 
Comparando-se com a verificação de vibrações ("galope") durante o trabalho, verificouse que estas ocorreram com intensidade considerável nos tratamentos D1P3 e D3P3, provocando nestes casos a interrupção dos ensaios. Estes tratamentos provocaram as maiores relações cinemáticas, 1,069 e 1,088, juntamente com o tratamento $\mathrm{D} 2 \mathrm{P} 3$, que, embora tendo $\mathrm{Kv}$ igual a 1,085 , não apresentou o "galope". O fato pode ser explicado com o auxílio da figura 1 , na qual os dois tratamentos relacionados apresentam altos $\mathrm{Kv}$ estáticos e relações entre patinamentos e as mais altas pressões nos pneus dianteiros. Este último parece ser um dos fatores que induzem o "galope", confirmando o que a maioria dos fabricantes, afirma quando recomendam diminuir a pressão interna dos pneus dianteiros sempre que ocorrer o fenômeno. No tratamento D2P1, em que havia uma relação cinemática estática negativa, não houve aparecimento do fenômeno, embora a relação entre patinamentos indicasse que isto devesse ocorrer. $\mathrm{Na}$ verdade, isso ocorreu por conta de um erro na medição de raios, comprovado pela relação entre raios. Nos outros tratamentos, foi aplicada uma relação cinemática entre os valores recomendados pela técnica $(1,02$ a 1,05$)$ não ocorrendo o aparecimento do fenômeno. A relação entre patinamentos era próxima a 1 , apresentando alta eficiência de tração e inexistência de "galope".

Desta forma, a relação cinemática calculada a partir dos raios estáticos serve como referência de projeto, baseada em raios sem carga, raios estáticos sob carga, raios índice informados por fabricantes. Estas referências têm muita utilidade também no desenvolvimento de estudos teóricos, como fizeram LINARES, (1996), SCHLOSSER,

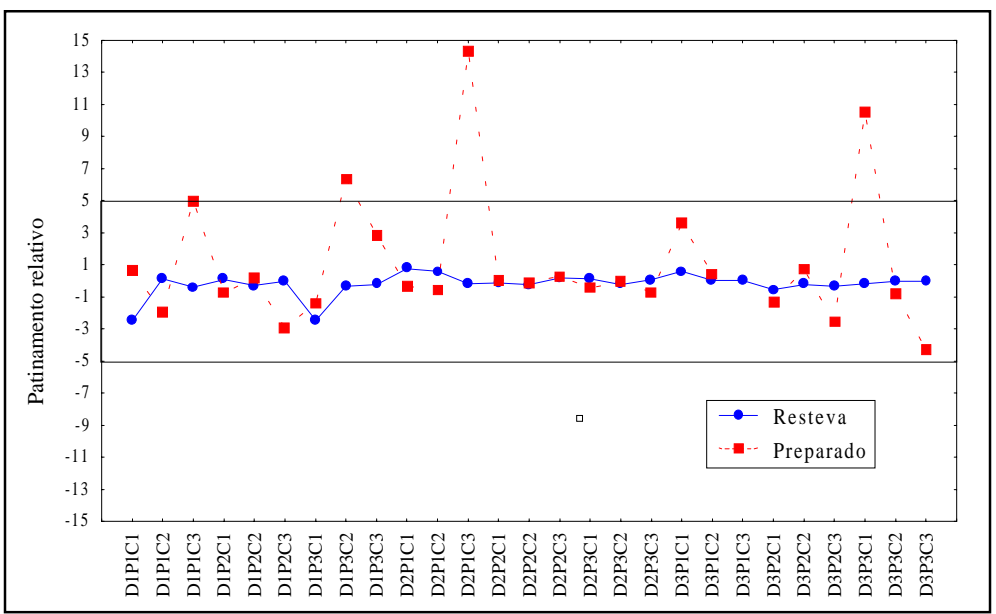

Figura 1 - Relação entre as velocidades das rodas obtidas a partir da medição com balizas.
(1996) e HERNÁNDEZ, (1999) e alguns autores que serviram de base para os trabalhos práticos que os seguiram.

A condição de referência tende a igualar todas as relações produzidas por combinações de pneus, pressões internas e transmissões do trator pois, como não há tração e os patinamentos são nulos ou muito baixos, as velocidades e distâncias desenvolvidas no campo pelos pneus nos dois eixos são muito semelhantes.

A condição dinâmica em trabalho de tração é a que realmente indica como está trabalhando o veículo para uma condição dada de solo, pois as rodas sofrem o efeito da tração, da resistência ao rolamento e o patinamento. $\mathrm{Na}$ condição dinâmica, independentemente do estado da superfície, o sistema solo-veículo, têm de absorver as diferenças entre as velocidades teóricas dos eixos. O solo se rompe parcialmente, e os pneus sofrem deformações submetidos a variações de carga e aderência produzindo quedas bruscas de ligação dos pneus com o solo de maneira alternada que provoca o fenômeno do "galope" do trator. Se a superfície do solo é dura, como em um caminho ou em resteva seca, os pneus que giram mais depressa se desgastam e suas garras se rompem para encurtar a sua circunferência. Em solo macio, como em solo úmido, comparável à situação de solo preparado, é o solo que fará o papel de fusível, permitindo o maior patinamento do pneu que gira mais rápido. $\mathrm{O}$ efeito da força de tração faz com que ocorra maior transferência de peso do eixo dianteiro ao traseiro, provocando maior coeficiente dinâmico de tração e aumento da sua eficiência de tração, mesmo que isto nem sempre se manifeste nos valores mais baixos de patinamento das rodas.

Pode-se calcular a relação entre as velocidades das rodas, multiplicandose a relação de raios pela relação entre velocidades angulares, e o patinamento relativo é o quociente entre $\mathrm{o}$ patinamento dianteiro e $\mathrm{o}$ traseiro, montando-se uma tabela que pode auxiliar na conclusão de que, a parte de dados erráticos, anteriormente detectados pela relação entre raios, ocorre sempre que a diferença percentual entre a relação de velocidades das rodas de referência e em tração alcance um valor de $\pm 5,0$ ou superior, o trator oscila e salta, realizando o efeito de "galope" (windup) detalhado na bibliografia por RACKHAM \& BLIGHT (1985). Nos ensaios de campo, comprovou-se esta afirmação, apontando-se tratamentos 
em que as oscilações eram fortes, a ponto de, em solo preparado, necessitar a parada do trator. Com a montagem desta tabela, pode-se verificar que as vibrações ocorrem quando a velocidade do eixo dianteiro é maior que a do traseiro. Quando um eixo gira mais rápido que o outro, haverá uma interferência cinemática e, na maioria das vezes, o eixo dianteiro é que tem de adaptar-se à velocidade do traseiro, para melhorar as condições de tração devido à transferência de peso e à maior área de contato do pneu com o solo.

\section{CONCLUSÕES}

O método utilizado para a determinação das condições de ocorrência do "galope" foi plenamente satisfatório, pois pôde prever com bastante precisão as condições em que iria ocorrer o fenômeno.

A utilização de altas cargas na barra de tração, combinada com elevadas pressões internas nos rodados dianteiros favoreceu o aparecimento do "galope".

Recomenda-se a diminuição gradativa das pressões internas dos pneus dianteiros, para evitar o aparecimento das vibrações.

Quando a diferença percentual entre a relação das velocidades teóricas de referência e da condição alcança um valor em torno de 5,0 ou superior produz-se o fenômeno conhecido como "galope" (power hop) do trator.

\section{REFERÊNCIAS BIBLIOGRÁFICAS}

BASHFORD, L.L. Axle power distribuition for a front-wheel assist trator. Transactions of the ASAE, v.28, n.5, p.13851388,1985

HERNÁNDEZ, P.M. Análisis del patinamiento en tractores de simple tracción a través de las teorias de predicción de tracción de ASAE y su contraste experimental. Espanha, 1999. Tese (Doutorado) - Universidad Politécnica de Madrid, 1999.

LINARES, P. Teoría de la tracción de tractores agrícolas. Madrid, 1996. 157p. Monografia (Escuela Técnica Superior de Ingeniros Agrónomos) - Escuela Técnica Superior de Ingenieros Agrónomos, Universidad Politécnica de Madrid, 1996.

RACKHAM, D.H., BLIGHT, D.P. Four-wheel drive trators - A review, Journal Agricultural Engeneering Research, Bedford, Inglaterra, v.2, p.185-201, 1985.

SCHLOSSER, J.F. Influencia del avance cinemático de las ruedas delanteras motoras en la influencia en tracción de los tractores agrícolas. Espanha, 1996. Tese (Doutorado) Universidad Politécnica de Madrid, 1996.

SRIVASTAVA, A.K., GOERING, C.E., ROHRBACH, R.P. Engineering principles of agricultural machines. $\mathrm{St}$ Joseph, USA : 1993. 601p. (ASAE textbook n.6).

Ciência Rural, v. 31, n. 6, 2001. 